\title{
OPTICAL SPECTRA OF LOW FLUX RADIO SOURCES
}

\author{
D. C. KOO \\ Lick Observatory, Board of Studies in Astronomy and Astrophysics \\ University of California, Santa Cruz, CA 95064 U.S.A. \\ R. G. KRON \\ Yerkes Observatory, University of Chicago \\ Williams Bay, WI 53191 U.S.A. \\ R. A. WINDHORST \\ Department of Physics, Arizona State University \\ Tempe, AZ 85281 U.S.A.
}

\section{Introduction}

Low flux radio sources are important for a variety of reasons. In principle, a $1 \mathrm{mJy}$ survey that is over 1000 times fainter than the $3 \mathrm{CR}$ allows us to reach objects 20 times more distant, almost literally to the edge of the universe, or to detect much less luminous sources at the same distances. We can hope to detect primeval galaxies, to check the idea that objects with redshifts $z$ greater than 3 or 4 are optically hidden by dust, to detect new populations of radio objects, to possess pointers to distant clusters and galaxies, as well as to understand better the AGN phenomenon.

The following highlights some of the optical findings associated with the LeidenBerkeley Radio Survey (LBDS). The initial $21 \mathrm{~cm}$ survey reached a $1 \mathrm{mJy}$ limit, covered $5.5 \mathrm{deg}^{2}$, and detected about 300 sources (Windhorst et al. 1984a). The optical data consist of multicolor $4 \mathrm{~m}$ plates (limit $\mathrm{B} \approx 24 \mathrm{mag}$ ), deep CCD frames (limit $\mathrm{B} \approx 26$ mag), and nearly 100 redshifts.

\section{Results}

- In contrast to the traditional view that most radio sources are either giant ellipticals or QSOs, our finding (Kron et al. 1985; Windhorst et al. 1987) is that low flux radio sources also consist of such diverse populations as ordinary stars, nuclei of spirals, probable supernovae events, peculiar/interacting galaxies with many having active star formation, and extended low surface brightness objects.

- The diversity in populations is reflected in the optical colors, which in the simplest form, can be divided between red galaxies probably similar to the traditional giant ellipticals, and blue galaxies, which include most of the remaining populations mentioned above. The reddest galaxies are noteworthy in being very similar in color to local giant ellipticals, even to redshifts of $\mathrm{z} \approx 0.8$, a result fully consistent with the findings of Hamilton (1985) for the reddest field galaxies and suggesting an old age for galaxies and the universe (Windhorst et al. 1986). Despite reaching much lower radio fluxes, almost all the red galaxies are optically quite luminous, 518 
similar to those found in the 3CR sample.

- At mJy and lower radio fluxes, the counts of radio sources increase more than expected (see e.g. Windhorst et al. 1985); the population contributing to this rise is dominated by blue galaxies, many of which have peculiar morphologies and often of very high radio and optical luminosities. Although the 21st mag blue objects among mJy sources were originally proposed to be distant giant ellipticals undergoing active star formation (Katgert et al. 1979), our redshifts suggest instead that none have redshifts beyond that expected for bright blue field galaxies with redshifts of several tenths.

- To the limits of $4 \mathrm{~m}$ plates, about $50 \%$ of the LBDS can be identified (Windhorst et al. 1984b). Pushing our optical identification work to fainter than 26th mag, we have been successful in identifying $100 \%$ of a subsample of 70 sources in one of our LBDS fields (Windhorst et al. 1987). This result implies either that faint radio sources do not extend beyond redshifts of 3 or 4 or that dust cannot totally block their optical counterparts.

- A major surprise in the faintest optical identifications was the presence of very low surface brightness extended objects, "fuzzballs", which are likely to be fainter, and perhaps higher redshift, counterparts to the few Lyman $\alpha$ clouds such as those already found among 3CR sources by McCarthy et al. (1987). We have attempted without success to secure redshifts for this important class of possible primeval galaxies.

- The luminosity function, nature, and evolution of the blue radio galaxies remain uncertain, especially since they may consist of several different classes of objects. The largest two redshifts so far among our radio galaxies are 1.32 and 2.39 , both large enough to require improvements to the radio evolution models originally proposed by Windhorst (1984).

\section{References}

Hamilton, D. 1985, Ap. J., 297, 371.

Katgert, P., de Ruiter, H. R., van der Laan, H. 1979, Nature, 280, 20.

Kron, R. G., Koo, D. C., and Windhorst, R. A. 1985, A.A., 146, 38.

McCarthy, P. J., Spinrad, H., Djorgovski, S., Strauss, M. A., van Breugel, W., and Liebert, J. 1987, Ap. J. (Letters), 319, L39.

Windhorst, R. A. 1984, Ph.D. Thesis, Leiden University.

Windhorst, R. A., Dressler, A., and Koo, D. C. 1987, IAU Symp. 124, Observational Cosmology, ed. A. Hewitt, G. Burbidge, and L. Z. Fang, p. 383.

Windhorst, R. A., Koo, D. C., and Spinrad, H. 1986, Proceedings of NATO Workshop on Galaxy Distances and Deviations from Universal Expansion, ed. B. F. Madore and R. B. Tully, p. 197.

Windhorst, R. A., van Heerde, G. M., Katgert, P. 1984a, A. A. Suppl., 58, 1.

Windhorst, R.A., Kron, R.G., and Koo, D. C. 1984b, A. A. Suppl., 58, 39.

Windhorst, R.A., Miley, G. K., Owen, F. N., Kron, R. G., Koo, D. C. 1985, Ap. J., 289, 494. 